\title{
Mobility Pattern Analysis for Power Restoration Activities Using Geo-Tagged Tweets
}

\author{
Bandana Kar* \\ karb@ornl.gov \\ Oak Ridge National Laboratory \\ Oak Ridge, TN, USA
}

\author{
Jacob Ethridge \\ jakeetheridge27@gmail.com \\ University of Tennessee \\ Chattanoogas, TN, USA
}

\begin{abstract}
In this study, we analyzed mobility patterns of at-risk populations affected by an extreme event using geo-tagged tweets to geo-target power restoration efforts. Unlike other studies that have used tweets to facilitate emergency management activities, we used 1.5 million geo-tagged tweets generated during Hurricane Sandy (2012) to determine the mobility patterns and geospatial distribution of impacted populations who experienced power outage before, during and after the hurricane. We implemented a three-step analytical framework to: (i) analyze tweet contents with visual methods, including dendrograms, word clouds to identify common keywords pertaining to power outage; (ii) identify target users whose tweets contained information about power outages; and (iii) create a user-tweet locations matrix and an origin-destination matrix to examine clusters of target users and their mobility patterns. Preliminary results indicate that potential clusters were present in and around New York city, Philadelphia, Washington D.C. and Baltimore, which were used as potential evacuation destination cities after hurricane Sandy. The travel pattern and destination information can be used to (i) mobilize restoration efforts by utility companies and (ii) address resource allocation needs both in impacted and destination cities. Future work will focus on analyzing potential destinations for different origins and travel-time to identify evacuation routing patterns.
\end{abstract}

\section{CCS CONCEPTS}

- Information systems $\rightarrow$ Content analysis and feature selection; Data analytics.

\section{KEYWORDS}

situational awareness, crisis informatics, mobility pattern analysis, power restoration, natural language processing

\section{ACM Reference Format:}

Bandana Kar and Jacob Ethridge. 2019. Mobility Pattern Analysis for Power Restoration Activities Using Geo-Tagged Tweets. In 2nd ACM SIGSPATIAL International Workshop on Advances on Resilient and Intelligent Cities (ARIC'19), November 5-8, 2019, Chicago, IL, USA. ACM, New York, NY, USA, 4 pages. https://doi.org/10.1145/3356395.3365547

\footnotetext{
*All authors contributed equally to this research.

Publication rights licensed to ACM. ACM acknowledges that this contribution was authored or co-authored by an employee, contractor or affiliate of the United States government. As such, the Government retains a nonexclusive, royalty-free right to publish or reproduce this article, or to allow others to do so, for Government purposes only.

ARIC'19, November 5-8, 2019, Chicago, IL, USA

(C) 2019 Copyright held by the owner/author(s). Publication rights licensed to ACM ACM ISBN 978-1-4503-6954-1/19/11 . \$ \$15.00

https://doi.org/10.1145/3356395.3365547
}

\section{INTRODUCTION}

While the threat of climate change grows, studies have indicated that the U.S. will experience frequent and severe tropical storms [5]. During 2017-2018, five tropical storms (hurricanes Michael, Florence, Harvey, Irma and Maria) impacted the U.S. causing about $\$ 325$ billion dollar loss and 3,269 deaths [13]. These events are a major contributor of large scale power outages in the U.S. due to damage to power infrastructures. In fact, since 2003, the number of weather related power outages have doubled [7], and these events can cost about $\$ 55$ billion annually [7]. They also contribute to mass evacuation and increased mobility from affected areas. This kind of abnormal mobility from disaster-prone areas contributes to resource allocation failures, road congestion, insufficient evacuation routing, and long-term response and recovery efforts as was seen in Puerto Rico following hurricane Maria.

Following a extreme event, emergency management measures are generally taken to mitigate adverse impacts of the event on social and physical environments, thereby preventing them from becoming disasters that far exceed impacted communities' coping capacity. Because social media and social network sites enable production of large volume of unstructured geo-spatial and nonspatial data before, during and after such events, these data have been used to undertake response and recovery efforts [2, 3, 14]. Among the many social media platforms, Twitter has been widely used for emergency management activities $[6,8,14]$.

Despite being noisy, the 140-character limit tweets used in this study contain fine-grained spatio-temporal data that is useful in gaining insight into the users' decision-making process in near real-time. This self-documented data allowed keyword analysis using hash-tags and meaningful keywords to differentiate tweets and analyze mobility pattern of users. Although only about $1.4-3 \%$ of tweets opt-in to share their location information that could be used for geo-tagging [10], the volume of tweets generated during hurricane Sandy (2012) made it plausible to use tweets in this study to examine the the mobility patterns and geo-spatial distributions of impacted populations before, during, and after hurricane Sandy.

This paper is organized into the following section. A brief overview of the hurricane Sandy related Twitter data set used in this study is presented in the next section. A discussion of the methodology implemented to determine mobility pattern is presented in section 3. Section 4 includes the preliminary results and discussion of the findings following which the conclusion and future work are presented in section 5 .

\section{JUSTIFICATION OF WORK}

Power restoration after an extreme event depends on extent of damage to power infrastructures, number of customers impacted, and 
availability of resources including crew members to dispatch for power restoration [9]. In many cases, the lack of such information can lead to long term outage as was the case during hurricane Maria (2017) in Puerto Rico. For faster recovery of impacted communities, several approaches are implemented to determine estimated time of restoration (ETR) $[12,16]$. However, ETR determination requires knowledge about outage prediction and/or actual outage information along with customers without power to estimate number of crews required for restoration [16]. Although utilities can provide information about degree and extent of power outages, failure of communication networks during extreme events can contribute to delayed information or no information about power outage, thereby hindering restoration process. Often there is no information available about power restoration.

An alternative is to use satellite-derived data that could be used independently or in conjunction with other sources to monitor restoration progress. Nightlight imagery such as the Visible Infrared Imaging Radiometer Suite (VIIRS) from NOAA that is available daily has been used to locate regions of blackout [15], but it requires clear weather conditions to capture information from satellites [4]. By contrast, tweets that contain location information as well as textual content pertaining to users' location, sentiment, etc. can be used to determine power outages $[4,11]$. Hultquist et al. [4], combined satellite imagery with geo-located tweets to determine areas of power outage at higher resolution than that of the night light imagery. The authors created a brightness change map using nightlight imagery and geo-tagged tweets filtered by keywords "power","blackout","electric","lights", "outage", "hurricane", "Sandy", and "storm" after hurricane Sandy. Likewise, Mao et al.[11] used keywords pertaining to power outages to target locations experiencing power outage in near real-time. A major takeaway of these studies is that geo-located tweets filtered by keywords can be used to identify damaged areas, areas experiencing power outage and areas where mass evacuation is occurring to help with emergency response efforts along with power restoration activities. Unlike previous studies, this study analyzed the movement pattern of impacted populations after hurricane Sandy who experienced power outage to identify potential origins and destinations. While the origin information could be used to geo-target restoration efforts, the origin-destination matrix can be used in conjunction with routing information for resource allocation. The framework developed here in can be implemented for other resource shortages, such as food and medical supplies.

\section{DATA AND METHODS}

Hurricane Sandy impacted regions of the northern Atlantic coast of the U.S. as it moved over the eastern coast until dissipating above Pennsylvania. We used the study site that spans between latitude $38^{\circ} \mathrm{N}-43^{\circ} \mathrm{N}$ and longitude $-73^{\circ} \mathrm{W}$ to $-78^{\circ} \mathrm{W}$ to extract tweets. This region is home to metropolitan areas that were affected by the storm, such as New York City, Washington D.C., Baltimore and Philadelphia.

In this preliminary study, we used more than 12 million tweets that were obtained for hurricane Sandy from [1]. Initial processing involved extracting the tweets that were in English language and dividing the extracted tweets into 24-hour chunks for the duration between October $22^{\text {nd }}$ and November $2^{\text {nd }}$. Specific attributes (e.g., tweet id, geo-coordinates, city and place names, tweet content, hash tags, and date/time) from the tweets for each day were extracted, which were used for analyses using $\mathrm{R}$. In addition to standard text cleaning, three filters using specific keywords were implemented to extract the tweets for analyses. The three filters used were: Off to identify impacted users (keywords: off, lost, without, donfit, have), Power to determine users experiencing power outage (keywords: power, outage, blackout, electric, nopower, darkness) and Sandy to determine users experiencing power outage due to hurricane Sandy (keywords: sandy, hurricane, hurricanesandy). After all the filters were implemented, 7,992 tweets $(0.0012 \%)$ were extracted for further analysis, which were generated within the U.S. (Table1). The userIDs from these tweets were used to analyze user movement.

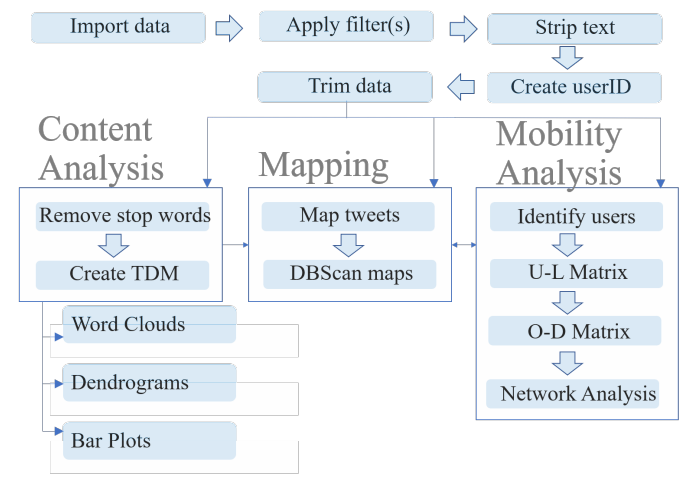

Figure 1: Framework To Create O-D Matrix

Figure 1 depicts the steps implemented to determine mobility pattern and create origin-destination matrix. First, a termDocumentMatrix was created, which was used to remove stop words and the word "power" due to its commonality across tweets. Wordclouds and cluster dendrograms were created for five days between Oct $28^{\text {th }}$-Nov $2^{\text {nd }}$ when maximum volume of tweets was generated. For dendrograms, the sparse was set to 0.94 to keep the dendrograms to 5-15 terms. Both dendrogram and wordcloud outputs were added to the original filters to create the final filter of suitable keywords. Using the filters, the targetUser group of tweets $(7,992)$ was divided into two groups: those experiencing power outage and those with power. The DBSCAN clustering approach was then implemented to identify the spatial clusters of tweets pertaining to power outage before, during and after the hurricane. Finally, for the targetUser group experiencing power outage, using usedID, user-location (U-L) matrix was created for each day for each user. This matrix included the potential destinations for each user over a $24 \mathrm{hr}$ period. Using the U-L matrix, origin-destination (O-D matrix) was created, which contained potential destinations for the same origin and the number of users traveling from the same origin to the same destination.

\section{RESULTS AND DISCUSSION}

Figure 2 displays the word cloud generated for October $31^{\text {st }}$. According to the word cloud, power is returning, which is expected as the hurricane had dissipated by then. Important keywords from the word cloud were "back" and "without", which helped differentiate between users with and without power after the hurricane. These keywords were used to determine the users the users who are no 


\begin{tabular}{|l|l|l|l|l|l|}
\hline Bin & $\begin{array}{l}\text { Total unfiltered } \\
\text { tweets }\end{array}$ & $\begin{array}{l}\text { Percent geo-tagged } \\
\text { tweets }\end{array}$ & $\begin{array}{l}\text { Percent geo-tagged tweets } \\
\text { with keyword power }\end{array}$ & $\begin{array}{l}\text { Percent geo-tagged tweets } \\
\text { with keyword sandy }\end{array}$ & $\begin{array}{l}\text { Percent tweets } \\
\text { with all filters }\end{array}$ \\
\hline Pre-Hurricane & 4045600 & $7.5 \%(304469)$ & $0.2 \%(673)$ & $0.3 \%(852)$ & NA \\
\hline Over-USA & 6715934 & $7.7 \%(514941)$ & $3.6 \%(18670)$ & $10.7 \%(54896)$ & $0.0012 \%(7992)$ \\
\hline Post-Hurricane & 2806817 & $6.2 \%(173656)$ & $2.4 \%(4169)$ & $8.6 \%(14867)$ & NA \\
\hline
\end{tabular}

Table 1: Percent Distribution of Tweets Before and After Hurricane Sandy and Over USA

longer experiencing power outage and identify those locations for further analyses.

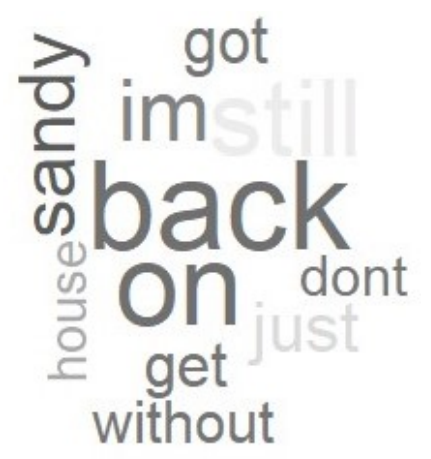

Figure 2: Word Cloud for October $3131^{\text {st }}$

According to Figure 3, during October $29^{\text {th }}-31^{\text {st }}$ (after landfall), there were four major clusters in New York City, Philadelphia, Baltimore, and Washington D.C. Figure 4 depicts the mobility pattern after the hurricane during Nov $1^{\text {st }}$ and Nov $5^{\text {th }}$. Again, most of the movement appears to be around these cities or in between metropolitan areas and suburbs. Given that New York City did experience significant storm surge, the concentration of large volume of tweets around the city is not surprising.

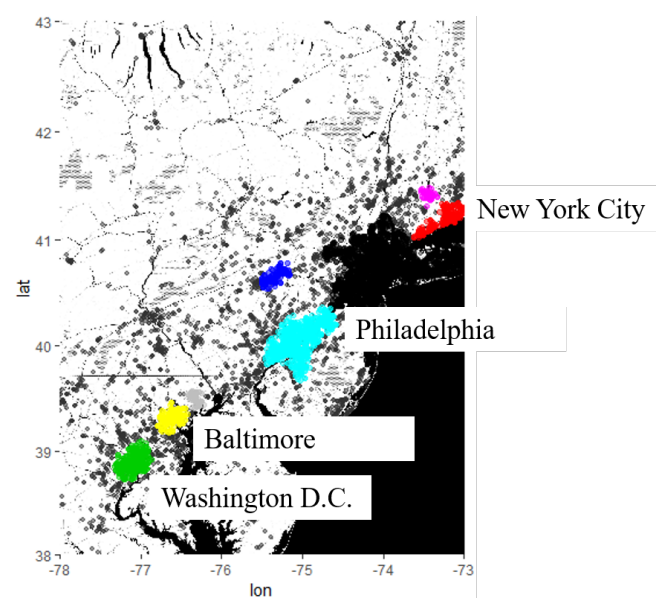

Figure 3: Mobility Map Based on User Clusters

Table 2 shows the potential locations traveled by users during Oct $27^{\text {th }}$-November $1^{\text {st }}$ according to the U-L matrix. The O-D matrix (Table 3) contains information about potential destinations for the

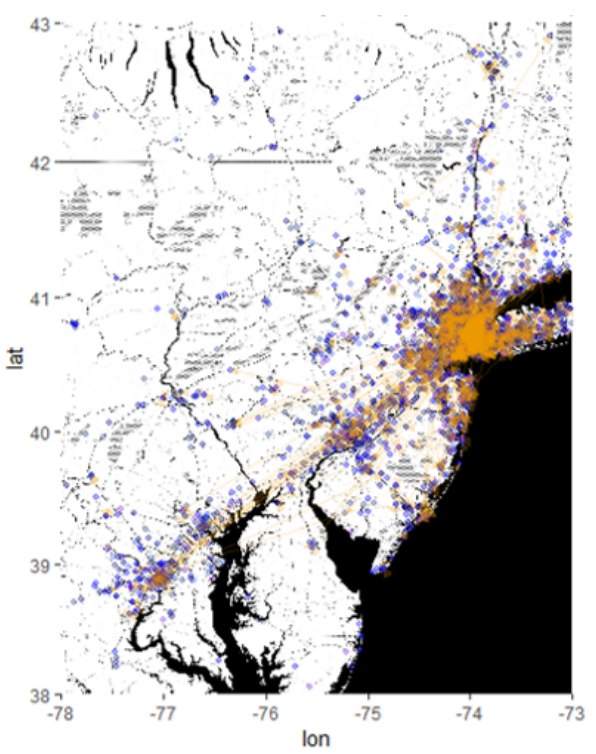

Figure 4: Mobility Clusters After Hurricane Landfall

same origin. According to table 3, there are 3 instances of a user traveling from Baltimore to Manhattan while there are 4 instances of a user traveling from Manhattan to Baltimore.

\section{CONCLUSION}

Evidently, tweets were effective in exploring mobility pattern. The matrix outputs of this study track users by day and organize user movements into readily usable matrices. Only around 18,000 tweets of the original sum of 1,464,748 geo-tagged tweets were eventually used in this study to create the matrix that included all tweets by targetUsers group. A wider group of targetUsers could be identified by (i) including non-geotagged tweets with keyword filters to find more impacted users and (ii) using separate keyword filters for other needs and resources (e.g. food, water, gas, shelter) to add users impacted by the hurricane but not specifically power outage. Future research using user-location matrix will be conducted to group users into regions around cities to study inter-city mobility due to Sandy for those impacted by power outage.

Majority of mobility occurred during and after the hurricane in and around New York city, Philadelphia, Washington D.C. and Baltimore. Many users stayed within the same area and conducted short-term trips to nearby cities as per the O-D matrix. The concentration of short-distance trips indicates that many people may be tweeting from home one day and then work the next day. However, it may also mean they are seeking shelter in nearby cities. Since 


\begin{tabular}{|l|l|l|l|l|l|l|}
\hline userID & $27-$ Oct & 28-Oct & 29-Oct & 30-Oct & 31-Oct & 1-Nov \\
\hline $2.52 \mathrm{E}+11$ & Dumont & Dumont & Dumont & Dumont & Dumont & Dumont \\
\hline $2.00 \mathrm{E}+11$ & Passaic & Passaic & Passaic & Passaic & Passaic & Passaic \\
\hline $1.60 \mathrm{E}+11$ & $\begin{array}{l}\text { New } \\
\text { Brunswick }\end{array}$ & $\begin{array}{l}\text { Old } \\
\text { Bridge }\end{array}$ & $\begin{array}{l}\text { New } \\
\text { Brunswick }\end{array}$ & NA & $\begin{array}{l}\text { New } \\
\text { Brunswick }\end{array}$ & $\begin{array}{l}\text { New } \\
\text { Brunswick }\end{array}$ \\
\hline $1.30 \mathrm{E}+11$ & NA & Kearny & Kearny & Kearny & Belleville & Kearny \\
\hline
\end{tabular}

Table 2: Sample User-Location Matrix

\begin{tabular}{|l|l|l|l|l|}
\hline & Baltimore & Manhattan & Philadelphia & Washington \\
\hline Baltimore (72) & 0 & 3 & 2 & 4 \\
\hline Manhattan (929) & 4 & 0 & 7 & 0 \\
\hline Philadelphia (1247) & 1 & 9 & 0 & 0 \\
\hline Washington (1674) & 4 & 0 & 0 & 0 \\
\hline
\end{tabular}

Table 3: Sample from O-D Matrix using four major cities

short-distance moves were far more common than long-distance moves, it was difficult to recognize a pattern in inter-region mobility other than that most long-distance moves were between major cities. Another limitation was that users tweeting about power outage from other events were difficult to discern from the actual impacted users. Also, there were likely many users who were not able to tweet because of power outage.

Nonetheless, the framework developed can be used to create $\mathrm{O}-\mathrm{D}$ matrix and analyze mobility pattern in conjunction with transportation and power outage data from reliable sources to determine potential travel routes and destination points. In future, network analysis will be conducted based on travel time in conjunction with impacted routes to determine if the potential destination is a shelter or workplace. Activity based intelligence can be obtained for the same users using tweets prior to the hurricane to identify potential destinations, which will help further classify the cluster types into shelters, hospitals, workplace, etc. Future research will also expand the location names to encompass regions so that commutes do not appear in the results. For example, one could group 50-mile radii around Baltimore and New York City to determine movement between those two regions. This could be done for all major cities identified by the clustering approach including Washington D.C and Philadelphia. Such results may be more useful in analyzing long-distance mobility.

\section{ACKNOWLEDGMENTS}

This manuscript has been authored by UT-Battelle, LLC under Contract No. DE-AC05-00OR22725 with the U.S. Department of Energy. The U.S. Government retains and the publisher, by accepting the article for publication, acknowledges that the U.S. Government retains a non-exclusive, paid-up, irrevocable, world-wide license to publish or reproduce the published form of this manuscript, or allow others to do so, for United States Government purposes.

\section{REFERENCES}

[1] Z. Arkaitz and H. Ji. 2013. Tweet, but Verify: Epistemic Study of Information Verification on Twitter. http://www.zubiaga.org/datasets/hurricane-sandy-tweets/. Social Network Analysis and Mining.
[2] J. Chan. 2012. How to Make Crowdsourcing Disaster Relief Work Better. US News and World Report.

[3] B. Heaton. 2013. How Emergency Managers can Benefit from Big Data. Emergency Management (2013).

[4] C. Hultquist, M. Simpson, G. Cervone, and Q. Huang. 2015. Using Nightlight Remote Sensing Imagery and Twitter Data to Study Power Outages. In Proceedings of the 1st ACM SIGNSPATIAL International Workshop on the Use of GIS in Emergency Management 2015.

[5] T. C. Richmond J. M. Melillo and G. W. Yohe. 2014. Climate Change Impacts in the United States: The Third National Climate Assessment. https://doi.org/10. 7930/J0Z31WJ2

[6] J. D. Kent and H. T. Capello. 2013. Spatial Patterns and Demographic Indicators of Effective Social Media Content During the Horsethief Canyon fire of 2012. Cartography and Geographic Information Science 40, 2 (2013), 78-89.

[7] A. Kenward and U. Raja. 2014. Blackout: Extreme Weather, Climate Change and Power Outages. Climate Central. http://assets.climatecentral.org/pdfs/ PowerOutages.pdf.Accessed14January2019.

[8] P. M. Landwehr and K. M. Carley. 2014. Social Media in Disaster Relief. In Data Mining and Knowledge Discovery for Big Data, W. W. Chu (Ed.). Springer, Berlin, 225-257.

[9] D. Lubkeman and D. E. Julian. 2004. Large Scale Storm Outage Management. IEEE Power Engineering Society General Meeting, 1-7.

[10] C. Nakos M. Malik, H. Lamba and J. Pfeffer. 2015. Population Bias in Geotagged Tweets. Association for the Advancement of Artificial Intelligence (2015).

[11] G. Mao, H.and Thakur, K. Sparks, J. Sanyal, and B. Bhaduri. 2018. Mapping NearReal-Time Power Outages from Social Media. International fournal of Digital Earth (2018). https://doi.org/1-15.10.1080/17538947.2018.1535000

[12] Guikema S. Nateghi, R. and S. M. Quiring. 2014. Power Outage Estimation for Tropical Cyclones: Improved Accuracy with Simpler Models. Risk Analysis (2014), 1069-1078.

[13] NOAA National Centers for Environmental Information (NCEI). 2019. U.S. Billion-Dollar Weather and Climate Disasters (2019). https://www.ncdc.noaa. gov/billions/.

[14] C. Pu and M. Kitsuregawa. 2014. Big Data and Disaster Management: A Report from the JST/NSF foint Workshop. JST/NSF Joint Workshop. https:/graitdm.gatech.edu/wp-content/uploads/2014/03/BigDataAndDisaster-v34.pdf.

[15] M. O. Romàn, E. C. Stokes, R. Shrestha, Z. Wang, L. Schultz, E. A. Sepùlveda Carlo, Q. Sun, J. Bell, A. Molthan, V. Kalb, C. Ji, K. C. Seto, S. McClain, and M. Enenkel. 2019. Satellite-Based Assessment of Electricity Restoration Efforts in Puerto Rico after Hurricane Maria. PLOS ONE (2019).

[16] T. Walsh, T. Layton, D. Wanik, and J. Mellor. 2018. Agent Based Model to Estimate Time to Restoration of Storm-Induced Power Outages. Infrastructures (2018), $1-21$. 